\title{
Clinical Impact of Adaptive Servo-Ventilation on the Left Atrial Pressure during Catheter Ablation in Sedated Patients with Atrial Fibrillation
}

Naohiko Kawaguchi $^{1}$, Atsushi Suzuki ${ }^{1}$, Michio Usui ${ }^{1}$, Shunji Yoshikawa ${ }^{1}$, Shingo

Watanabe $^{1}$, Ryota Maeno ${ }^{1}$, Hirofumi Kujiraoka ${ }^{1}$, Kuniyoshi Sato ${ }^{1}$, Masahiko Goya ${ }^{2}$, and

Tetsuo Sasano ${ }^{3}$

${ }^{1}$ Tokyo Yamate Medical Center

${ }^{2}$ Tokyo Medical and Dental University Hospital

${ }^{3}$ Tokyo Medical and Dental University

October 5, 2020

\begin{abstract}
Introduction Sedation during pulmonary vein isolation (PVI) of atrial fibrillation often provokes a decline in left atrial (LA) pressure (LAP) under atmospheric pressure and increases the risk of systemic air embolisms. This study aimed to investigate the efficacy of adaptive servo-ventilation (ASV) on the LAP in sedated patients. Methods and Results Fifty-one consecutive patients undergoing cryoballoon PVI were enrolled. All patients underwent sedation using propofol throughout the procedure. Sedation status was monitored by the bispectral index. After the transseptal puncture and inserting the long sheath into the LA, the LAP was measured via the sheath. Then, the ASV treatment was started, and the LAP was re-measured. The LAP before and after the ASV support was investigated. Before the ASV, the LAP during inspiratory phase was significantly smaller than that during expiratory phase $(4.9 \pm 5.4 \mathrm{mmHg}$ vs. $14.0 \pm 5.2 \mathrm{mmHg}, \mathrm{p}<0.01)$. The lowest LAP was $-2.2 \pm 5.1 \mathrm{mmHg}$ and was under $0 \mathrm{mmHg}$ in $37(73 \%)$ patients. After the ASV, the LAP during inspiratory phase significantly increased to $8.9 \pm 4.1$ $\mathrm{mmHg}(\mathrm{p}<0.01)$, and lowest LAP to $4.7 \pm 5.9 \mathrm{mmHg}(\mathrm{p}<0.01)$. The negative lowest LAP value became positive in $30 / 37(81 \%)$ patients. There were no statistical differences regarding obstructive sleep apnea (OSA), obesity, gender, or other comorbidities between patients with and without a negative lowest LAP after the ASV support. Conclusion ASV is effective for increasing the LAP above $0 \mathrm{mmHg}$ and might prevent air embolisms during PVI. A negative LAP after the ASV was rare but occurred in patients even without comorbidities such as OSA and obesity
\end{abstract}

Clinical Impact of Adaptive Servo-Ventilation on the Left Atrial Pressure during Catheter Ablation in Sedated Patients with Atrial Fibrillation

Naohiko Kawaguchi, MD*, Atsushi Suzuki, MD*, Michio Usui, MD*, Shunji Yoshikawa, MD*, Shingo Watanabe, MD*, Ryota Maeno, MD*, Hirofumi Kujiraoka, MD*, Kuniyoshi Sato, MD*, Masahiko Goya, MD ${ }^{+}$, Tetsuo Sasano, $\mathrm{MD}^{+}$

*Heart Center, Tokyo Yamate Medical Center, Tokyo, Japan

${ }^{+}$Department of Cardiology, Tokyo Medical and Dental University, Tokyo, Japan.

Address for correspondence: Atsushi Suzuki

3-22-1 Hyakunin-cho, Shinjuku-ward, Tokyo, Japan, 169-0073

Heart Center, Tokyo Yamate Medical Center, Tokyo, Japan 
Telephone: +81-03-3364-0251

Fax: +81-03-3364-5663

E-mail: bellwood@msc.biglobe.ne.jp

Disclosures: None

\section{Abstract}

\section{Introduction}

Sedation during pulmonary vein isolation (PVI) of atrial fibrillation often provokes a decline in left atrial (LA) pressure (LAP) under atmospheric pressure and increases the risk of systemic air embolisms. This study aimed to investigate the efficacy of adaptive servo-ventilation (ASV) on the LAP in sedated patients.

\section{Methods and Results}

Fifty-one consecutive patients undergoing cryoballoon PVI were enrolled. All patients underwent sedation using propofol throughout the procedure. Sedation status was monitored by the bispectral index. After the transseptal puncture and inserting the long sheath into the LA, the LAP was measured via the sheath. Then, the ASV treatment was started, and the LAP was re-measured. The LAP before and after the ASV support was investigated.

Before the ASV, the LAP during inspiratory phase was significantly smaller than that during expiratory phase $(4.9 \pm 5.4 \mathrm{mmHg}$ vs. $14.0 \pm 5.2 \mathrm{mmHg}, \mathrm{p}<0.01)$. The lowest LAP was $-2.2 \pm 5.1 \mathrm{mmHg}$ and was under $0 \mathrm{mmHg}$ in $37(73 \%)$ patients. After the ASV, the LAP during inspiratory phase significantly increased to $8.9 \pm 4.1 \mathrm{mmHg}(\mathrm{p}<0.01)$, and lowest LAP to $4.7 \pm 5.9 \mathrm{mmHg}(\mathrm{p}<0.01)$. The negative lowest LAP value became positive in $30 / 37(81 \%)$ patients. There were no statistical differences regarding obstructive sleep apnea (OSA), obesity, gender, or other comorbidities between patients with and without a negative lowest LAP after the ASV support.

\section{Conclusion}

ASV is effective for increasing the LAP above $0 \mathrm{mmHg}$ and might prevent air embolisms during PVI. A negative LAP after the ASV was rare but occurred in patients even without comorbidities such as OSA and obesity.

Key words: ablation, adaptive servo ventilation, atrial fibrillation, left atrial pressure, sedation

\section{Introduction}

Pulmonary vein isolation (PVI) is a widely accepted procedure for catheter ablation of patients with atrial fibrillation (AF). For a completion of the PVI, access to the left atrium (LA) is required, and a transseptal puncture has usually been performed. The procedure in the LA has a substantial risk of systemic embolisms including cerebral and myocardial infarctions ${ }^{1-2}$.

The PVI using a cryoballoon has emerged as an alternative ablation procedure tool for radiofrequency (RF) ablation and its safety and effectiveness have been $\operatorname{proven}^{3-4}$. However, thrombi and air embolic events during cryoballoon ablation have recently been reported ${ }^{2,5}$. In terms of air embolic events during the procedure, the creation of procedure related micro air bubbles has been thought to be the most common factor ${ }^{6-7}$. The majority of micro air embolisms are silent, asymptomatic, and detected by brain magnetic resonance imaging after the procedure ${ }^{6,8}$. On the other hand, a large amount of an air embolism during the catheter ablation procedure is rare, but could be life-threatening ${ }^{1-2}$. It is mainly caused by massive air intrusion through a long sheath located in the LA and is prone to occur at the time the intra LA pressure (LAP) drops to less than the atmospheric pressure ${ }^{1,9}$.

Patients typically receive sedation during the PVI because the procedures take several hours and often are inflictive. Sedation of patients could inevitably result in an upper airway obstruction and subsequently 
provoke apnea and snoring, which could reduce the intra thoracic pressure and LAP. A decreased LAP to less than the atmospheric pressure increases the risk of air intrusion into the vasculature through a long sheath.

Adaptive servo-ventilation (ASV) (AutoSet CS; ResMed, Sydney) is a noninvasive positive pressure ventilation and is designed to treat sleep-disordered breathing ${ }^{10-11}$. It provides a positive expiratory airway pressure and inspiratory pressure support, and automatically adjusts the airway ventilation volume based on the analysis of the patient's breathing effort. Although the efficacy of the ASV therapy in patients with sleep-disordered breathing has been widely accepted, the accurate efficacy of ASV for the LAP in sedated patients remains unclear.

The aim of this study was to investigate the LAP in sedated patients undergoing a cryoballoon PVI and to clarify the impact of the ASV on the LAP during the procedure.

\section{Methods}

\section{Inclusion and exclusion criteria}

From October 2018 to September 2020, a cryoballoon PVI was performed in patients with drug refractory AF. All patients received sedation and a subsequent ASV treatment was applied during the procedure. Consecutive patients undergoing a cryoballoon PVI with ASV support under sedation were enrolled in this study. The LAPs before and after the ASV support were sequentially evaluated in all these patients.

Exclusion criteria were severe valvular disease, hemodialysis, severe coronary artery disease, a LA diameter $>55 \mathrm{~mm}$, and the existence of a thrombus in the LA.

Written informed consent was obtained from all patients. This study protocol was approved by the institutional review board of our institute and conformed to the ethical guidelines of the 1975 Declaration of Helsinki.

\section{Procedural management}

All patients received oral anticoagulants prior to the procedure. In patients taking direct oral anticoagulants, the drugs were interrupted on the day of the procedure and re-administered on the next morning after the procedure. In patients taking warfarin, the warfarin therapy was continued without interruption.

During the procedure, a single transseptal puncture was performed with an RF needle (Baylis Medical Inc, Montreal, Quebec, Canada) and 8 Fr long sheath (SL0, Abbott, Minneapolis, MN). Five thousand U of Heparin was administered before the transseptal puncture and additionally given to maintain an activated clotting time between 300 and 350 seconds. After the transseptal puncture, an 8 Fr long sheath was exchanged for a steerable $15 \mathrm{Fr}$ sheath (Flexcath Advance, Medtronic, Minneapolis, MN).

\section{The LAP evaluation and ASV support}

The atmospheric pressure was set as $0 \mathrm{mmHg}$ reference at a beginning of the procedure. After the transseptal puncture and insertion of the $15 \mathrm{Fr}$ long sheath (FlexCath) in the LA, the LAP via the sheath was recorded on a digital polygraph (RMC-5000, Nihon Corden, Tokyo, Japan) (Figure 1 ). The LAP was measured over more than three consecutive respiratory cycles. The LAP during one respiratory cycle was calculated as the average of the integral LAPs during one respiratory cycle, and the LAP was defined as the average value of that over three or more consecutive cycles. The LAP during the inspiratory and expiratory phases also was defined in the same manner. The lowest LAP was the minimum LAP during those respiratory cycles. Afterward, the ASV treatment was initiated in all patients. As the setting for the ASV, an expiratory positive airway pressure of $5 \mathrm{cmH}_{2} \mathrm{O}$ and inspiratory pressure support between 3 and $10 \mathrm{cmH}_{2} \mathrm{O}$ were used. Following the ASV treatment and confirmation of stable breathing synchronized with the ASV, the LAP was re-measured.

\section{Sedation}


All patients received sedation throughout the procedure. At the beginning of the procedure, an intravenous propofol bolus of $0.5 \mathrm{mg} / \mathrm{kg}$ was administered, and a nasopharyngeal temperature probe was inserted and located in the esophagus to measure the esophageal temperature during the procedure. Maintenance of sedation was performed with a continuous intravenous propofol administration with a dosage of $1.25^{\sim} 2$ $\mathrm{mg} / \mathrm{kg} / \mathrm{hr}$. As an analgesic, an intravenous drip infusion of buprenorphine with a dosage of $1.0 \mu \mathrm{g} / \mathrm{kg}$ was also administered at the beginning of the procedure and was properly added if patients complained of pain. The bispectral index was continuously monitored by a BIS monitoring system (A-3000 BIS XP Platform, Aspect Medical Systems, Newton, MA) to evaluate the sedation level of the patients. To maintain the BIS index level between 50 and 70 , an intravenous propofol bolus of $0.5 \mathrm{mg} / \mathrm{kg}$ was additionally administered, and the dosage of the continuous intervenous propofol administration was also adjusted according to the sedation level of the patients, if necessary ${ }^{12-13}$.

\section{Ablation procedure}

The method of the cryoballoon ablation has been previously described ${ }^{8}$. In brief, a steerable 15 Fr sheath (Flexcath) and second-generation 28mm cryoballoon (Arctic Front Advance, Medtronic) were inserted into the LA and advanced to the orifice of the targeted PVs. Then, the cryoballoon PVI was started. The entire PVI procedure was performed under ASV support.

\section{Statistical analysis}

Continuous data are expressed as the mean \pm SD. Statistical comparisons were performed using the Student's t-test and Fisher exact test, as appropriate. Statistical significance was set at a value of $\mathrm{P}<0.05$. Statistical analysis was performed using Bell curve for Excel (ver. 3.20).

\section{Results}

\section{Study population and baseline patient characteristics}

Fifty-one consecutive patients underwent the cryoballoon PVI. The mean age was $65.8 \pm 10.4$ years old and $41(80.4 \%)$ patients had a male gender. The proportion of paroxysmal and non-paroxysmal AF was 27 (53.0 $\%)$ and $24(47.0 \%)$ patients, respectively. Two patients were diagnosed with obstructive sleep apnea (OSA) and were introduced to continuous positive airway pressure therapy. The patient characteristics are shown in Table 1 .

\section{LAP evaluation (Table 2 )}

The LAPs were sequentially measured before and after the ASV support in a total of 51 enrolled patients. The BIS value at the time of the LAP measurement was similar between that before and after the ASV support $(67.7 \pm 13.1$ vs. $68.5 \pm 12.7, \mathrm{p}=0.26)$. Out of 27 patients with paroxysmal AF, the LAP was measured during sinus rhythm in 25 and during AF in the remaining 2. In all 24 patients with non-paroxysmal AF, the LAP was measured during AF or atrial flutter. There was no significant difference in the average LAP between that before and after the ASV support $(10.9 \pm 4.8 \mathrm{mmHg}$ vs. $11.6 \pm 3.7 \mathrm{mmHg}, \mathrm{p}=0.14)$.

\section{LAP before the ASV}

The LAP during the inspiratory phase was $4.9 \pm 5.4 \mathrm{mmHg}$ and was significantly lower than that during the expiratory phase $(14.0 \pm 5.2 \mathrm{mmHg}, \mathrm{p}<0.01)$. The average lowest LAP was $-2.2 \pm 5.1 \mathrm{mmHg}$ (from 7 to $-17 \mathrm{mmHg}$ ), which was recorded during the inspiratory phase in all patients. Out of a total of 51 patients enrolled, $37(72.5 \%)$ had a lowest LAP value of [?] $0 \mathrm{mmHg}$. A male gender was frequently observed in patients with a lowest LAP value of [?] $0 \mathrm{mmHg}$ (negative lowest LAP) than in those with a lowest LAP value of $>0 \mathrm{mmHg}(33 / 37$ vs. $8 / 14, \mathrm{p}=0.02)$. There were no significant differences regarding the BIS index, body-mass index (BMI), prevalence of OSA, obesity (BMI $>25 \mathrm{~kg} / \mathrm{m} 2$ ), and other comorbidities between the patients with and without a negative lowest LAP value (Table 3A ). The BIS value at the time of the LAP measurement was similar between the two groups $(67.4+-12.5$ vs. $68.7+-14.4, \mathrm{p}=0.54)$.

\section{LAP after the ASV}


After the ASV, the LAP during the inspiratory phase significantly increased to $8.9+-4.1 \mathrm{mmHg}$ as compared to that before the ASV $(4.9+-5.4 \mathrm{mmHg})(\mathrm{p}<0.01)$ (Figure 2$)$. The $\Delta$ lowest LAP between that before and after the ASV support was $6.6 \pm 5.2 \mathrm{mmHg}$. The lowest LAP increased in $47 / 51(92.2 \%)$ patients with the ASV, and remained unchanged in the other four patients (Figure 3 ). Out of 37 patients with a negative lowest LAP value before the ASV, in $30(81.1 \%)$ patients the lowest LAP value turned out to be positive after the ASV. Even in the remaining seven patients, the lowest LAP increased to $4.6 \pm 3.5 \mathrm{mmHg}$, but it did not reach more than $0 \mathrm{mmHg}$ (from -6 to $0 \mathrm{mmHg}$ ). There were no significant differences regarding the patient characteristics or comorbidities between the patients with and without a negative lowest LAP value after the ASV (Table 3B ). The BIS value at the time of the LAP measurement was similar between the two groups $(65.0 \pm 13.6$ vs. $69.0 \pm 12.5, \mathrm{p}=0.75)$. The male gender also was not observed very frequently in the patients with a negative lowest LAP value $(7 / 7$ vs. $34 / 41, \mathrm{p}=0.32)$.

\section{Expiratory LAP evaluation}

After the ASV, the expiratory LAP slightly decreased from $14.0 \pm 5.2 \mathrm{mmHg}$ to $12.7 \pm 4.3 \mathrm{mmHg}(\mathrm{p}<0.01)$. The $\Delta$ expiratory LAP difference between that before and after the ASV support was $-1.3 \pm 3.3 \mathrm{mmHg}$.

\section{Results of the cryoballoon PVI}

Out of a total of 204 enrolled PVs, all PVs were successfully isolated. Although temporary right phrenic nerve paralysis was observed in two patients during the cryoballoon ablation at the RSPV, the motion of the diaphragm fully recovered by the first out patient visit. A groin hematoma was observed in three patients. There were no other complications during the procedure including systemic embolic events such as cerebral and myocardial infarctions.

\section{Discussion}

The major findings in the present study were (1) the lowest LAP in sedated patients dropped to less than the atmospheric pressure in approximately $73 \%$ of patients, (2) ASV increased the lowest LAP value to positive in $81 \%$ of the patients who had a negative lowest LAP value before the ASV support, (3) there were no significant differences regarding the patient characteristics including the prevalence of OSA, BMI, and a male gender between the patients with and without a negative lowest LAP value after the ASV support.

A drop in the LAP of less than the atmosphere pressure during the PVI increases the risk of massive air intrusion during the procedure. The hemostasis valve of the sheath plays an important role in the prevention of air intrusion into the vasculature through a long sheath. However, the valve could become open to the atmosphere while inserting a catheter into the sheath via the valve, especially when inserting catheters with complicated tip shapes such as circular mapping catheters or multipolar catheters ${ }^{2}{ }^{6}$. If the timing of the insertion of the catheter is synchronized with a drop in the LAP to less than atmospheric pressure, a massive air embolism is prone to occur. The continuous maintenance of the LAP above the atmospheric pressure during the procedure could absolutely decrease the risk of air intrusion. The present study confirmed that the majority of the sedated patients undergoing AF ablation had a negative LAP during the procedure, and to the best of our knowledge, this is the first clinical study that has demonstrated the efficacy of the ASV in the prevention of an LAP drop to less than the atmospheric pressure. Although the present study evaluated the LAP in patients undergoing a cryoballoon PVI, this result could be applicable to all kinds of percutaneous catheter procedures which require access into the LA.

In addition to providing a supply of positive expiratory pressure, ASV has a unique algorithm that automatically supplies positive inspiratory pressure according to an analysis of the patient's breathing effort. It could provide a stable respiration pattern. Although bronchial intubation could certainly more effectively reduce the risk of air intrusion and contribute to a stable LAP, it is invasive and requires general anesthesia, however, the ASV treatment is easy to start and finish. Therefore, an evaluation of the impact of ASV on the LAP could be worthwhile.

\section{AF ablation with sedation}


The feasibility and safety of deep sedation during AF ablation has been previously reported ${ }^{14-15}$. However, intravenous sedation with an anesthetic agent such as propofol causes respiratory suppression due to upper airway collapse, and the anesthetic depth is associated with the respiratory status ${ }^{16}$.

As the sedation depth, anesthetic dosing was adjusted to maintain the BIS value within a target range of 50-70 and actual average BIS value before the ASV support was approximately 68, suggesting the sedation depth was nearly moderate sedation ${ }^{12-13}$. In patients under the moderate sedation depth, it is generally considered that no interventions are required to maintain a patent airway and spontaneous ventilation is adequate $^{17}$. However, the present study revealed that the $73 \%$ of the sedated patients had negative lowest LAP value before the ASV support. That suggested that the LAP could become negative even in patients under moderate sedation. During the procedure, the sedation level often resulted in a deeper sedation level than initially intended. Therefore, the fact that the risk of air intrusion might change depending on the sedation continuum should be noted.

Additionally, the introduction and maintenance of the sedation was performed with an intravenous propofol administration. For an intravenous anesthetic agent during AF ablation, propofol, midazolam, dexmedetomidine are commonly used. Out of those anesthetic agents, dexmedetomidine has a relatively less propensity for the induction of an upper air way collapse in comparison to propofol ${ }^{12}$. If dexmedetomidine is administered for the anesthesia, the prevalence of a negative LAP under sedation could be smaller.

\section{Evaluation of negative lowest LAP even after the ASV support}

The present study revealed the ability of the ASV treatment to increase the LAP above the atmospheric pressure. On the other hand, the lowest LAP was still negative in seven patients even with ASV support. Previous reports demonstrated that OSA has been associated with an LAP decline during sleep ${ }^{18}$. Furthermore, obesity, a male gender, hypertension, diabetes mellitus, and heart failure are thought to be important risk factors for $\mathrm{OSA}^{19-20}$. However, those specific patient characteristics could not frequently be observed in patients with a negative LAP after the ASV. In the present study, there were two patients who had been diagnosed with OSA before the ablation procedure, and that might have influenced the results.

We should be aware that a negative LAP after the ASV could be provoked even in patients without OSA, obesity, or other risk factors. Complications due to massive air intrusion could be fatal, and therefore, not only the efficacy but the limitations of ASV on the LAP must be taken into consideration. If further study reveals and predicts the characteristics of the patients with a high risk of an ASV refractory negative LAP, selective and prophylactic bronchial intubation before the procedure could be effective in preventing air intrusion.

\section{Expiratory LAP}

In the patients under sedation, the inspiratory LAP was easily influenced by a decline in the intra thoracic pressure, however, the expiratory LAP theoretically would not. Therefore, the expiratory LAP in patients under sedation was thought to be almost identical to the LAP in those in an awake state.

The expiratory LAPs after the ASV were slightly lower than those before the ASV. From the aspect of the mechanism of noninvasive positive pressure ventilation, a positive expiratory pressure might reduce the venosus return and decreased the LAP. However, whether a positive expiratory pressure influences the LAP immediately after the initiation of the ASV remains unclear and requires further investigation in terms of the hemodynamic status.

\section{Study limitations}

This study was a single center trial and the study population was relatively small. The LAP was not evaluated continuously because the insertion of the cryoballoon into the long sheath was needed to perform the PVI and an accurate LAP could not be evaluated during cryoballoon ablation. The sedation status was variable even with the same dose of an anesthetic agent, and the collapsibility of the upper airway also changed during the procedure. An additional propofol bolus administration as the patient status dictated easily deepen the 
sedation status for a certain length of time. Therefore, the efficacy of the ASV for the LAP could be variable and labile.

The present study did not perform polysomnography to evaluate the extent of the OSA before the ablation procedure. Therefore, the prevalence of OSA might have been underestimated. Further prospective studies that enroll larger numbers of patients with OSA and investigate the LAP change after the ASV treatment are expected.

\section{Conclusion}

The ASV treatment in sedated patients undergoing a PVI was effective for increasing the LAP above the atmospheric pressure and might prevent fatal air embolisms during the procedure. Furthermore, the LAP could drop and become negative in patients under sedation even in those without OSA, obesity, or other comorbidities.

\section{References}

1. Kuwahara T, Takahashi A, Takahashi Y, Kobori A, Miyazaki S, Takei A, Fujii A, Kusa S, Yagishita A, Okubo K, Fujino T, Nozato T, Hikita H, Sato A, Aonuma K. Clinical characteristics of massive air embolism complicating left atrial ablation of atrial fibrillation: lessons from five cases. Europace 2012, $14(2), 204-8$.

2. Cay S, Ozeke O, Ozcan F, Topaloglu S, Aras D. Coronary air embolism during cryoablation of atrial fibrillation: A catastrophic complication and its management. J Atr Fibrillation 2017 Oct 31; 10 (3): 1728.

3. Aryana A, Singh SM, Kowalski M, Pujara DK, Cohen AI, Singh SK, Aleong RG, Banker RS, Fuenzalida CE, Prager NA, Bowers MR, D'Avila A, O'Neill PG. Acute and long-term outcomes of catheter ablation of atrial fibrillation using the second-generation cryoballoon versus open-irrigated radiofrequency: a multicenter experience. J Cardiovasc Electrophysiol 2015, 26 (8), 832-839.

4. Kuck KH, Brugada J, Furnkranz A, Metzner A, Ouyang F, Chun KR, Elvan A, Arentz T, Bestehorn K, Pocock SJ, Albenque JP, Tondo C. Cryoballoon or radiofrequency ablation for paroxysmal atrial fibrillation. N Engl J Med 2016, 374 (23), 2235-45.

5. Tsukahara K, Oginosawa Y, Fujino Y, Ohe H, Yamagishi Y, Iwataki M, Sonoda S, Kohno R, Otsuji Y. Abe H. Prevention of serious air embolism during cryoballoon ablation; risk assessment of air intrusion into the sheath by catheter selection and change in intrathoracic pressure: an ex vivo study. J Cardiovasc Electrophysiol 2019, 30 (12), 2944-2949.

6. Miyazaki S, Watanabe T, Kajiyama T, Iwasawa J, Ichijo S, Nakamura H, Taniguchi H, Hirao K, Iesaka Y. Thromboembolic risks of the procedural process in second-generation cryoballoon ablation procedures: analysis from real-time transcranial Doppler Monitoring. Circ Arrhythm Electrophysiol 2017, 10 (12).

7. Takami M, Fujiwara R, Kijima Y, Nagoshi R, Kozuki A, Mochizuki Y, Shibata H, Nakano S, Fukuyama Y, Fujimoto D, Kakizaki S, Masuko E, Kurimoto H, Kyo S, Miyata T, Shite J. Techniques for reducing air bubble intrusion into the left atrium during radiofrequency catheter and cryoballoon ablation procedures: An ex vivo study with a high-resolution camera. Heart Rhythm 2019, 16 (1), 128-139.

8. Nakamura T, Okishige K, Kanazawa T, Yamashita M, Kawaguchi N, Kato N, Aoyagi H, Yamauchi Y, Sasano T, Hirao K. Incidence of silent cerebral infarctions after catheter ablation of atrial fibrillation utilizing the second-generation cryoballoon. Europace 2017, 19 (10), 1681-1688.

9. Franzen OW, Klemm H, Hamann F, Koschyk D, von Kodolitsch Y, Weil J, Meinertz T, Baldus S. Mechanisms underlying air aspiration in patients undergoing left atrial catheterization. Catheter Cardiovasc Interv 2008, 71 (4), 553-8.

10. Miyata M, Yoshihisa A, Suzuki S, Yamada S, Kamioka M, Kamiyama Y, Yamaki T, Sugimoto K, Kunii H, Nakazato K, Suzuki H, Saitoh S, Takeishi Y. Adaptive servo ventilation improves Cheyne-Stokes respiration, cardiac function, and prognosis in chronic heart failure patients with cardiac resynchronization therapy. J Cardiol 2012, 60 (3), 222-7.

11. Randerath WJ, Galetke W, Stieglitz S, Laumanns C, Schafer T. Adaptive servo-ventilation in patients 
with coexisting obstructive sleep apnoea/hypopnoea and Cheyne-Stokes respiration. Sleep Med 2008, $9(8), 823-30$.

12. Kasuya Y, Govinda R, Rauch S, Mascha EJ, Sessler DI, Turan A. The correlation between bispectral index and observational sedation scale in volunteers sedated with dexmedetomidine and propofol. Anesth Analg 2009, 109 (6), 1811-5.

13. Wang ZH, Chen H, Yang YL, Shi ZH, Guo QH, Li YW, Sun LP, Qiao W, Zhou GH, Yu RG, Yin K, He $\mathrm{X}, \mathrm{Xu}$ M, Brochard LJ, Zhou JX. Bispectral index can reliably detect deep sedation in mechanically ventilated patients: a prospective multicenter validation study. Anesth Analg 2017, 125 (1), $176-183$.

14. Kottkamp H, Hindricks G, Eitel C, Muller K, Siedziako A, Koch J, Anastasiou-Nana M, Varounis C, Arya A, Sommer P, Gaspar T, Piorkowski C, Dagres N. Deep sedation for catheter ablation of atrial fibrillation: a prospective study in 650 consecutive patients. J Cardiovasc Electrophysiol 2011, 22 (12), 1339-43.

15. Salukhe TV, Willems S, Drewitz I, Steven D, Hoffmann BA, Heitmann K, Rostock T. Propofol sedation administered by cardiologists without assisted ventilation for long cardiac interventions: an assessment of 1000 consecutive patients undergoing atrial fibrillation ablation. Europace 2012, 14 (3), 325-30.

16. Eastwood PR, Platt PR, Shepherd K, Maddison K, Hillman DR. Collapsibility of the upper airway at different concentrations of propofol anesthesia. Anesthesiology. 2005 Sep; 103 (3): 470-7.

17. Practice guidelines for moderate procedural sedation and analgesia 2018: A report by the American society of anesthesiologists task force on moderate procedural sedation and analgesia, the American association of oral and maxillofacial surgeons, American college of radiology, American dental association, American society of dentist anesthesiologists, and society of interventional radiology. Anesthesiology. 2018 Mar; 128 (3): 437-479.

18. Condos WR Jr, Latham RD, Hoadley SR, Pasipoularides A. Hemodynamics of the Mueller maneuver in man: right and left heart micromanometry and doppler echocardiography. Circulation. 1987 Nov; 76 (5): 1020-8.

19. Heinzer R, Vat S, Marques-Vidal P, Marti-Soler H, Andries D, Tobback N, Mooser V, Preisig M, Malhotra A, Waever G, Vollenweider P, Tafti M, Haba-Rubio J. Prevalence of sleep-disordered breathing in the general population: the HypnoLaus study. Lancet Respir Med. 2015 Apr; 3 (4): 310-8.

20. Nieto FJ, Young TB, Lind BK, Shahar E, Samet JM, Redline S, D’Agostino RB, Newman AB, Lebowitz MD, Pickering TG. Association of sleep-disordered breathing sleep apnea and hypertension in a large community based-study. Sleep Heart Health Study. JAMA. 2000 Apr 12; 283 (14): 1829-36.

Table 1 : Clinical characteristics of the study population

\begin{tabular}{ll}
\hline Number of patients, $\mathrm{n}$ & 51 \\
\hline Age, years & $65.8 \pm 10.4$ \\
Male gender (\%) & $41(80.4)$ \\
$\mathrm{BMI}^{+}, \mathrm{kg} / \mathrm{m} 2$ & $24.4 \pm 3.6$ \\
Obesity (BMI > 25 kg/m2) (\%) & $20(39)$ \\
Obstructive sleep apnea (\%) & $2(3.9)$ \\
Non-paroxysmal AF ${ }^{*}(\%)$ & $24(47.0)$ \\
CHADS2 score & $1.0 \pm 0.8$ \\
Hypertension (\%) & $25(49.0)$ \\
Diabetes mellitus (\%) & $6(11.8)$ \\
Heart failure (\%) & $8(15.7)$ \\
Old cerebral infarction and TIA ${ }^{+}(\%)$ & $2(3.9)$ \\
BNP on the day before ABL, pg/mL & $95.9 \pm 141.7$ \\
LA ${ }^{\S}$ diameter, mm & $41.2 \pm 7.1$ \\
LVEF ${ }^{\mathbb{I}, \%}$ & $62.1 \pm 8.9$ \\
\hline
\end{tabular}

${ }^{*}$ AF: atrial fibrillation 
${ }^{+}$BMI: body-mass index

$\S_{\text {LA: left atrium }}$

ILVEF: left ventricular ejection fraction

+TIA: transient ischemic attack

Table 2 : Comparison of the LAP and BIS value between that before and after the ASV support

\begin{tabular}{llll}
\hline & Before the $\mathrm{ASV}^{*}$ & After the ASV & $\mathrm{p}$ value \\
\hline Average $\mathrm{LAP}^{+}, \mathrm{mmHg}$ & $10.9 \pm 4.8$ & $11.6 \pm 3.7$ & 0.14 \\
LAP during the inspiratory phase, $\mathrm{mmHg}$ & $4.9 \pm 5.4$ & $8.9 \pm 4.1$ & $<0.01$ \\
Lowest LAP, mmHg & $-2.2 \pm 5.1$ & $4.7 \pm 5.9$ & $<0.01$ \\
Number of lowest LAPs [?] 0 mmHg (\%) & $37(72.5)$ & $7(13.7)$ & $<0.01$ \\
LAP during the expiratory phase, mmHg & $14.0 \pm 5.2$ & $12.7 \pm 4.3$ & $<0.01$ \\
BIS value at the time of the LAP measurement & $67.7 \pm 13.1$ & $68.5 \pm 12.7$ & 0.26 \\
\hline
\end{tabular}

*ASV: Adaptive Servo-Ventilation

+ LAP: left atrial pressure

Table 3 : Comparison of the characteristics between the patients with and without a negative lowest LAP value

Before the ASV support

\begin{tabular}{llll}
\hline & Lowest LAP [?] & Lowest LAP $>0$ & p value \\
\hline Number of patients, $\mathrm{n}$ & 37 & 14 & \\
Age, years & $66.8 \pm 9.9$ & $63.3 \pm 3.9$ & 0.37 \\
Male gender (\%) & $33(89)$ & $8(36)$ & 0.02 \\
BMI $^{+}, \mathrm{kg} / \mathrm{m} 2$ & $24.2 \pm 2.7$ & $24.8 \pm 3.9$ & 0.84 \\
Obesity (BMI > 25 kg/m2) (\%) & $15(41)$ & $5(36)$ & 1.0 \\
Obstructive sleep apnea (\%) & $2(5)$ & $0(0)$ & 1.0 \\
Non-Paroxysmal AF ${ }^{*}(\%)$ & $19(51)$ & $5(36)$ & 0.36 \\
CHADS2 score & $1.03 \pm 0.75$ & $0.93 \pm 0.96$ & 0.57 \\
Hypertension (\%) & $19(51)$ & $6(43)$ & 0.55 \\
Diabetes mellitus (\%) & $5(14)$ & $1(7)$ & 1.0 \\
Heart failure (\%) & $5(14)$ & $3(21)$ & 0.67 \\
Old cerebral infarction and TIA ${ }^{+}(\%)$ & $1(3)$ & $1(7)$ & 0.48 \\
BNP, pg/mL & $87 \pm 144.3$ & $120.6 \pm 132.7$ & 0.75 \\
LA $A^{\S}$ diameter, mm & $41.0 \pm 7.3$ & $42.2 \pm 6.4$ & 0.70 \\
LVEF $^{\mathbb{I}, \%}$ & $62.3 \pm 9.6$ & $61.6 \pm 6.4$ & 0.43 \\
\hline
\end{tabular}

(B) After the ASV support

\begin{tabular}{llll}
\hline & Lowest LAP [?] & Lowest LAP $>0$ & $\mathrm{p}$ value \\
\hline Number of patients, $\mathrm{n}$ & 7 & 44 & \\
Age, years & $69.0 \pm 13.9$ & $65.3 \pm 9.6$ & 0.85 \\
Male gender (\%) & $7(100)$ & $34(77)$ & 0.32 \\
$\mathrm{BMI}^{+}, \mathrm{kg} / \mathrm{m} 2$ & $24.6 \pm 4.9$ & $24.3 \pm 3.3$ & 0.47
\end{tabular}




\begin{tabular}{llll}
\hline & Lowest LAP [?] & Lowest LAP $>0$ & $\mathrm{p}$ value \\
\hline Obesity (BMI > 25 kg/m2) $(\%)$ & $4(57)$ & $16(36)$ & 0.41 \\
Obstructive sleep apnea (\%) & $1(14)$ & $1(2)$ & 0.20 \\
Non-Paroxysmal AF ${ }^{*}(\%)$ & $4(57)$ & $20(45)$ & 0.69 \\
CHADS2 score & $1.1 \pm 0.8$ & $0.98 \pm 0.8$ & 0.66 \\
Hypertension (\%) & $3(43)$ & $22(50)$ & 1.0 \\
Diabetes mellitus (\%) & $2(29)$ & $4(9)$ & 0.19 \\
Heart failure (\%) & $1(14)$ & $7(16)$ & 1.0 \\
Old cerebral infarction and TIA $^{+}(\%)$ & $0(0)$ & $2(5)$ & 1.0 \\
BNP, pg/mL & $86.1 \pm 262.2$ & $97.6 \pm 92.9$ & 0.35 \\
LA diameter, mm & $42.3 \pm 6.9$ & $41.0 \pm 7.1$ & 0.44 \\
LVEF $\mathbb{I}, \%$ & $64.6 \pm 11.7$ & $61.7 \pm 8.3$ & 0.84 \\
\hline
\end{tabular}

${ }^{*} \mathrm{AF}$ : atrial fibrillation

+BMI: body-mass index

${ }^{\S} \mathrm{LA}$ : left atrium

$\mathbb{I}_{\text {LVEF: left ventricular ejection fraction }}$

+TIA: transient ischemic attack

\section{Figure legends}

Figure 1: The LAP waveform

Respiratory variations in the LAP waveform during the procedure were observed, and the inspiratory LAP prominently dropped to less than the atmospheric pressure $(0 \mathrm{mmHg})$.

The average LAP, that during the inspiratory and expiratory phase, and the lowest LAP were measured, respectively.

Exp: expiratory phase

Insp: inspiratory phase

LAP: left atrial pressure 


\section{Figure 1}

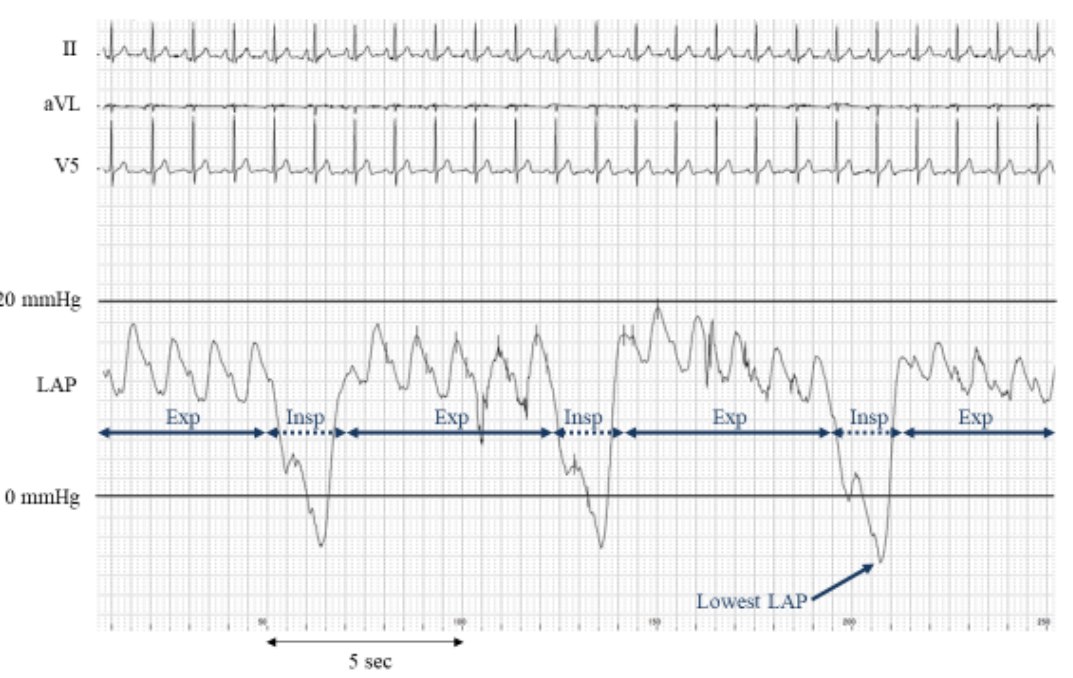

Figure 2 : Representative case of the LAP between that before (A) and after (B) the ASV support. The steep decline in the LAP during the inspiratory phase completely resolved and the lowest LAP increased to a positive value after the ASV support.

ASV: Adaptive Servo-Ventilation

LAP: left atrial pressure

Figure 2(A)

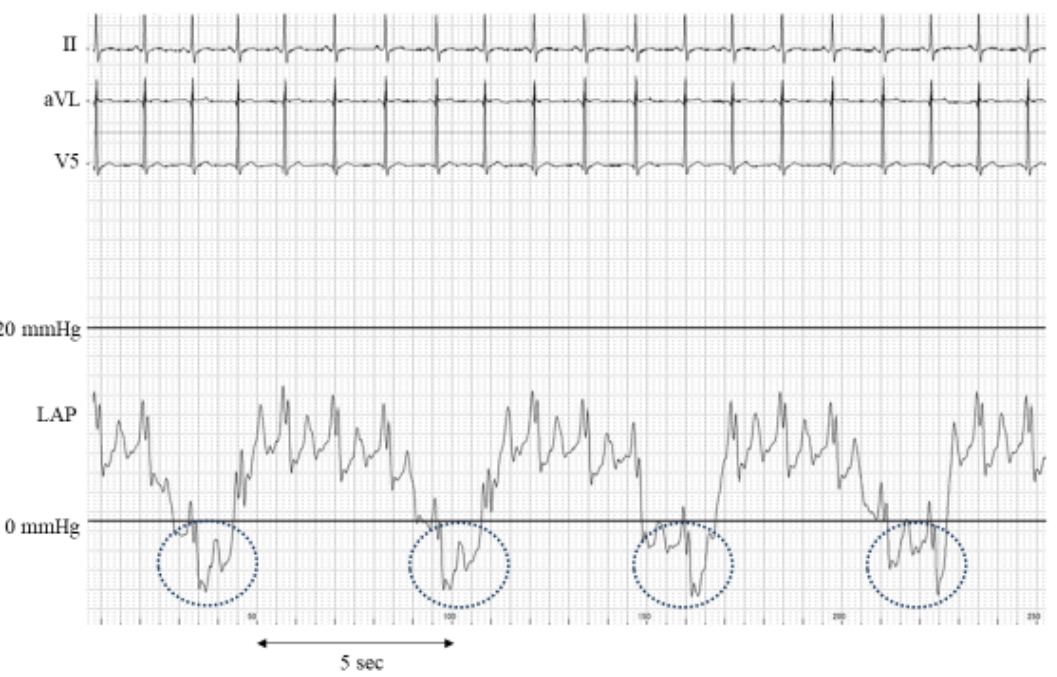




\section{Figure 2(B)}

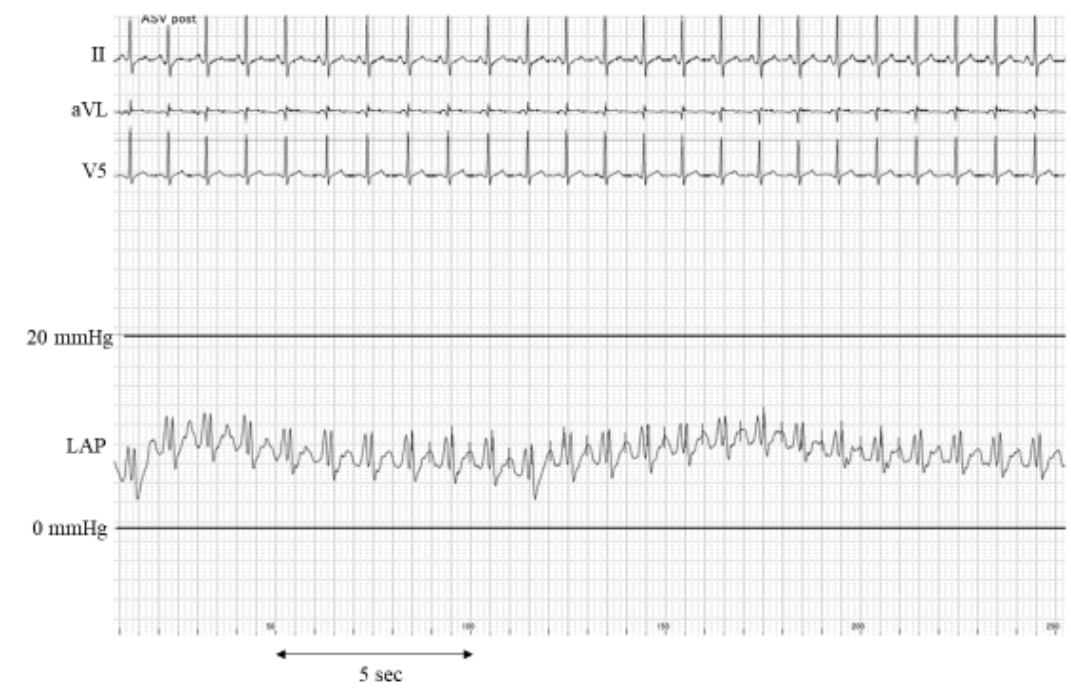

Figure 3 : Change in the lowest LAP between that before and after the ASV support ASV: Adaptive Servo-Ventilation

LAP: left atrial pressure

Figure 3

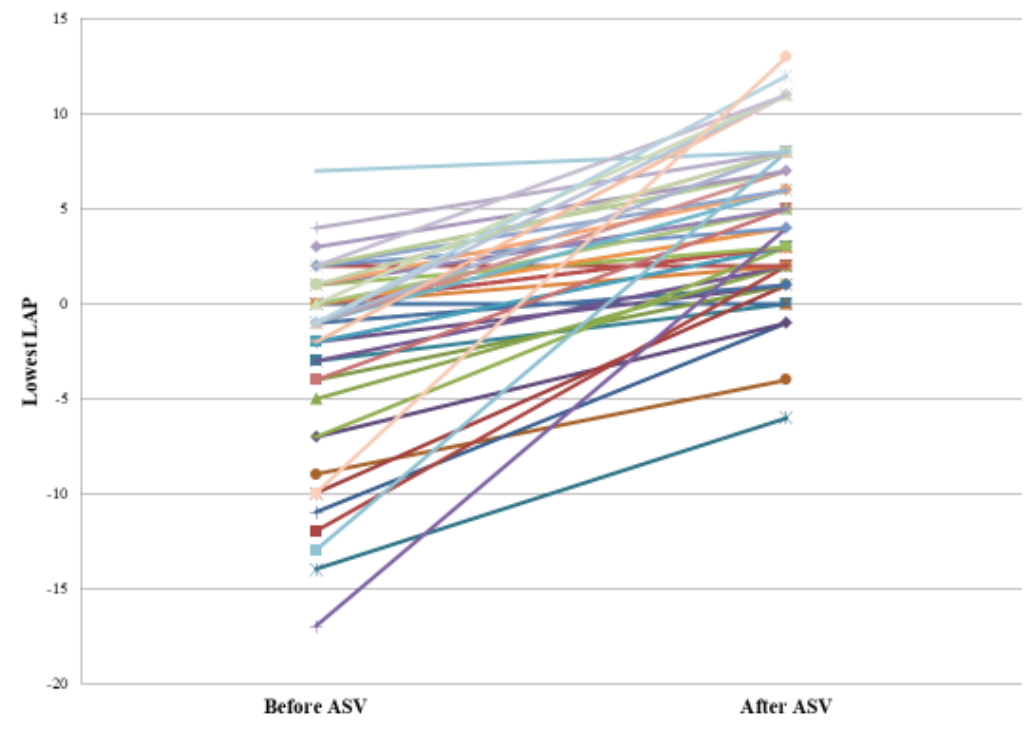


Figure 1

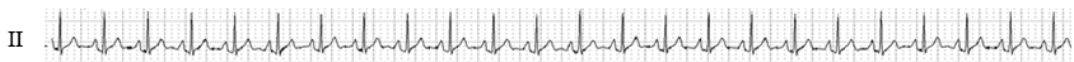

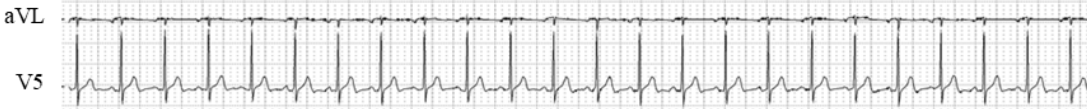

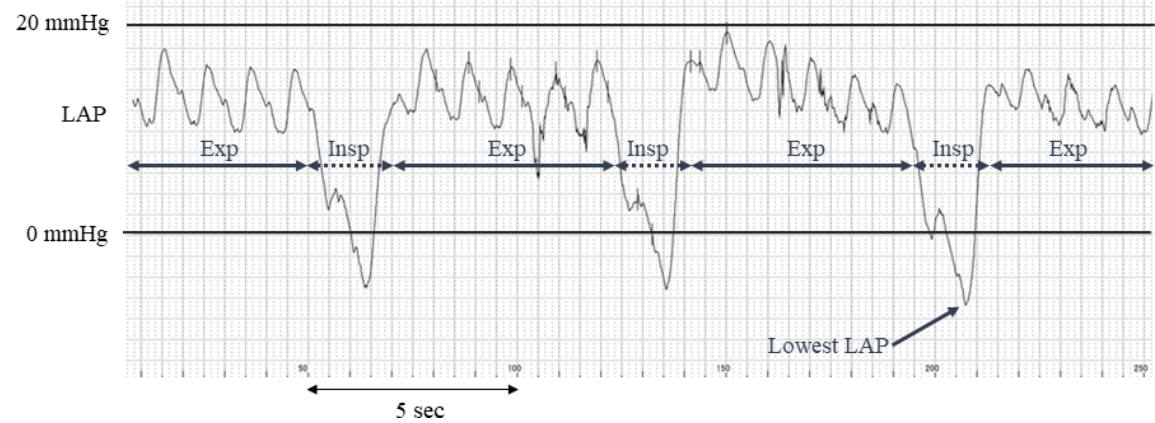

Figure 2(A)

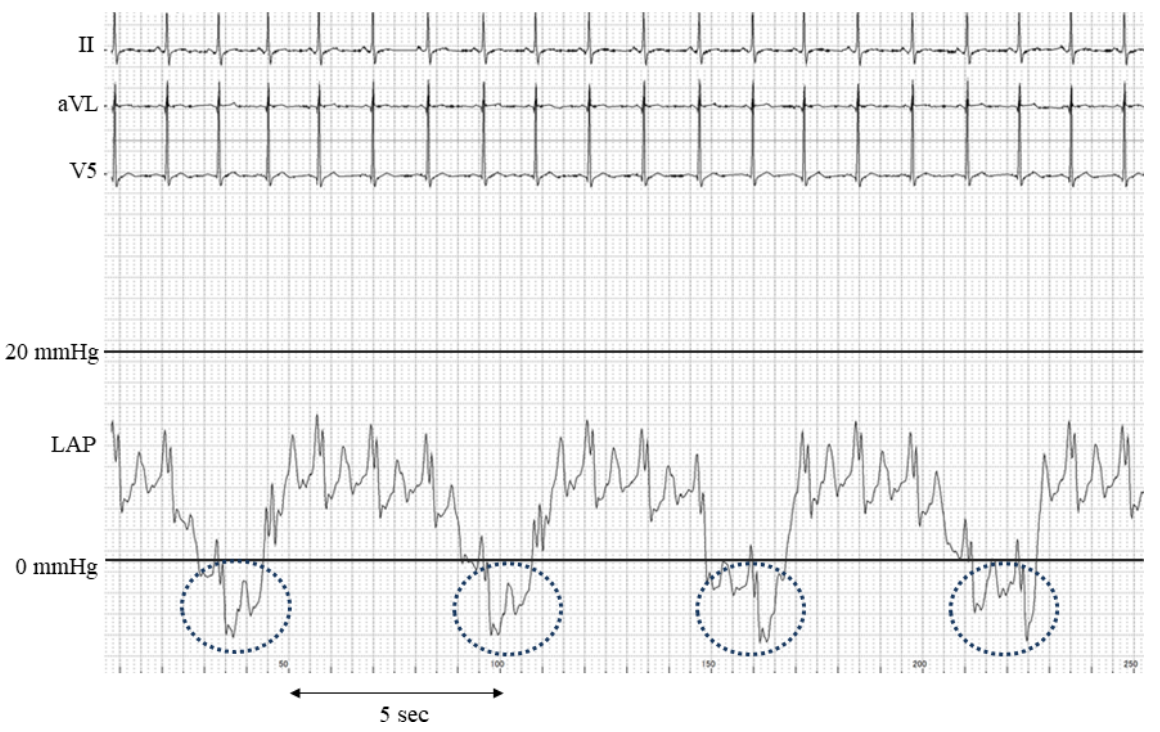


Figure 2(B)

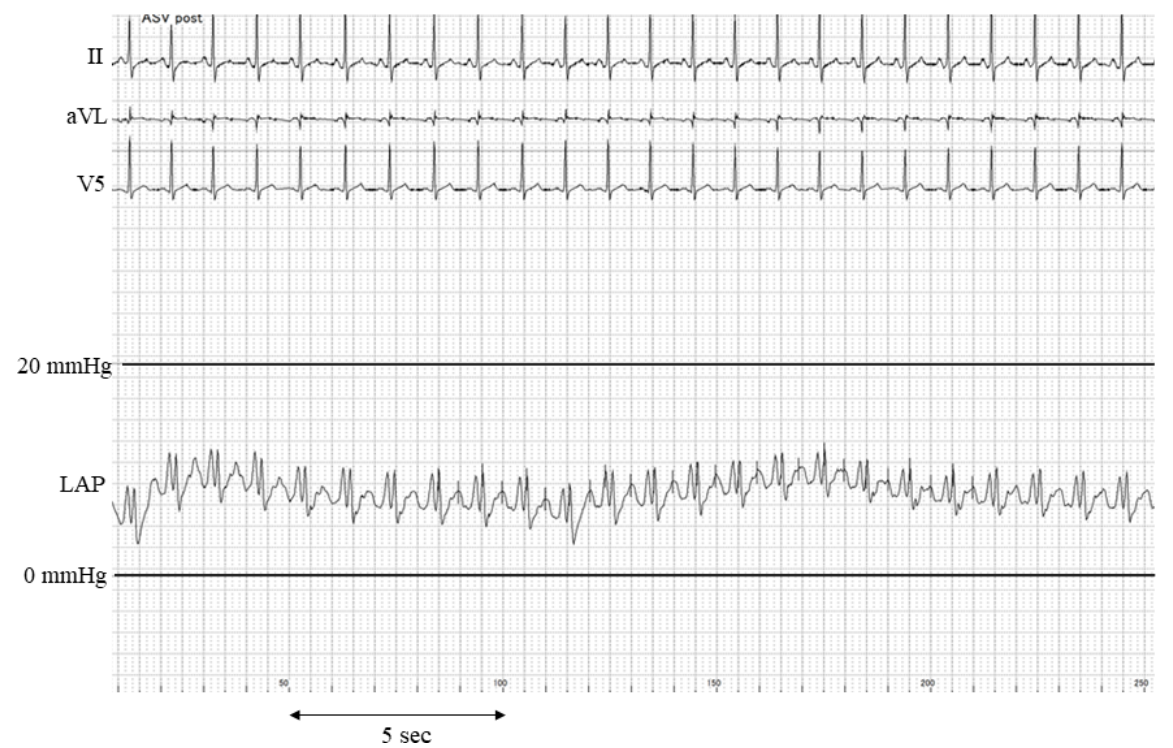

Figure 3

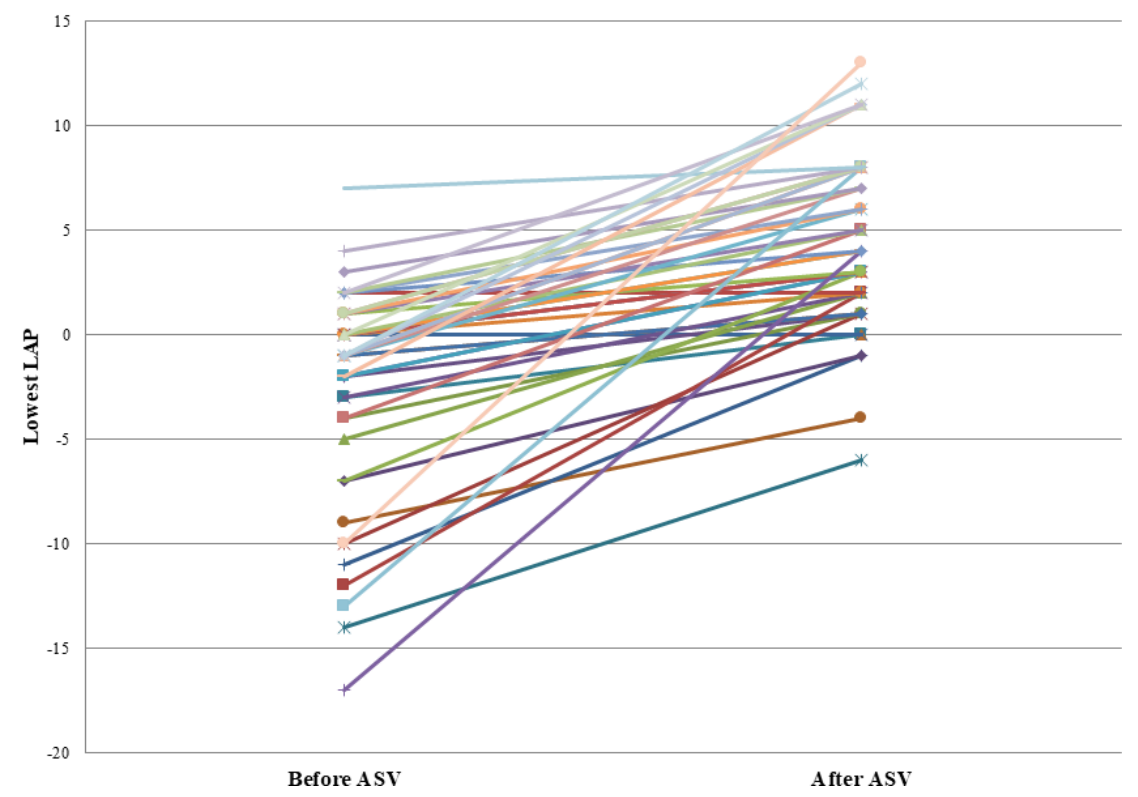

